\title{
Carpal Tunnel Syndrome in Patients on Intermittent Hemodialyis
}

\author{
Helmut Schiffl \\ Department of Medicine IV, University of Munich, Munich, Germany
}

Busch et al. [1] report a high prevalence of carpal tunnel syndrome (CTS) in hemodialysis (HD) patients in spite of the use of high-flux dialyzer membranes and ultrapure dialysis fluid. The article is of interest as dialysis-related amyloidosis (DRA) has been out of focus in the last decade.

The interpretation of the data presented is, however, hampered by the disadvantages of the cross-sectional study design they chose.

(1) One major pitfall of this descriptive study is the absence of a clear, specific and reproducible disease definition. The gold standard for the diagnosis of DRA manifestations remains the immunohistological detection of amyloid. Clinical assessment of symptoms and signs may pave the way to the diagnosis, but do not prove the presence of amyloid deposits in CTS. The authors do not differentiate between different causes (diabetes) of CTS nor grade the severity of destructive arthropathy, which may lead to overdiagnosis. The authors found a prevalence of CTS of $28.7 \%$ in HD patients with a dialysis vintage of

\section{References}

1 Busch M, Schwenzky A, Franke S, Stein G, Wolf G: Advanced glycation end products and $\beta_{2}$-microglobulin as predictors of carpal tunnel syndrome in hemodialysis patients. Blood Purif 2012;34:3-9.

2 Koch KM: Dialysis-related amyloidosis. Kidney Int 1992;41:1416-1429.

3 Kuchle C, Fricke H, Held E, Schiffl H: Highflux hemodialysis postpones clinical manifestation of dialysis-related amyloidosis. Am J Nephrol 1996;16:484-488. less than 60.3 months. By comparison, other authors reported the prevalence of CTS as 0 at 5 years [2] when low-flux membranes and commercial (potentially contaminated) dialysis fluid were used. Busch et al. [1] clearly overestimated the prevalence of CTS in their patients (median follow-up 31 months).

(2) Cross-sectional studies may establish associations, but not causality. Due to the nonrandomized design, confounders may be unequally distributed among subgroups. Indeed, in the study by Busch et al. [1], patients treated with high-flux biocompatible dialyzer membranes had the highest dialysis duration, the lowest residual diuresis, and the highest circulating $\beta_{2}$-microglobulin concentrations. Moreover, the use of high-flux membranes was associated with a higher prevalence of CTS in the multivariate logistic regression analysis. These data are in contrast to other reports demonstrating lower $\beta_{2}$-microglobulin levels in high-flux HD compared to low-flux HD of similar HD vintage and a lower prevalence of CTS in these patients $[3,4]$.
(3) Cross-sectional studies ascertain exposure and outcome at the same time. There is convincing evidence that the microbiological quality of the dialysis fluid is an important determinant of DRA [5]. Unfortunately, the authors were not able to analyze the impact of the dialysis fluid purity on the prevalence of CTS. They had no information on this variable when their patients were dialysed before their investigations had started.

Our retrospective analysis of comparable HD patient groups (1995-2010, a total of 147 patients, dialysis vintage at least 10 years) undergoing predominantly (more than $75 \%$ of all sessions) either low-flux HD utilising commercial dialysis fluid or receiving ultrapure high-flux HD revealed a decrease in histologically proven CTS from 68 to $28 \%$ (unpublished data). This trend is in line with other studies $[6,7]$ reporting a decreased prevalence of CTS over time. We agree with Busch et al. [1] that DRA remains an unsolved problem of long-term maintenance HD.
4 Schiffl H, Fischer R, Lang SM, Mangel E: Clinical manifestations of $\mathrm{AB}$-amyloidosis: effects of biocompatibility and flux. Nephrol Dial Transplant 2000;15:840-845.

5 Baz M, Durand C, Ragon A, Jaber K, Andrieu D, Merzouk T, Purgus R, Olmer M, Reynier JP, Berland Y: Using ultrapure water in hemodialysis delays carpal tunnel syndrome. Int J Artif Organs 1991;14:681-685.
6 Schwalbe S, Holzhauer M, Schaeffer J, Galanski M, Koch KM, Floege J: Beta 2-microglobulin associated amyloidosis: a vanishing complication of long-term hemodialysis? Kidney Int 1997;52:1077-1083.

7 Cianciolo G, Coli L, La MG, Donati G, D’Addio F, Comai G, Ricci D, Dormi A, Wratten M, Feliciangeli G, Stefoni S: Is beta2-microglobulin-related amyloidosis of hemodialysis patients a multifactorial disease? A new pathogenetic approach. Int J Artif Organs 2007;30:864-878.

\section{KARGER}

Fax +4161306 1234 E-Mail karger@karger.ch www.karger.com
Prof. Helmut Schiffl

University of Munich, Department of Medicine IV

Ziemssenstrasse 1

DE-80336 Munich (Germany)

E-Mailh-schiffl@t-online.de 


\title{
Reply
}

\author{
Martin Busch, MD, Günter Stein, MD, Gunter Wolf, MD \\ Department of Internal Medicine III, Jena University Hospital, Friedrich-Schiller-University, Jena, Germany
}

We thank Dr. Helmut Schiffl and colleagues for their comments on our study [1]. We absolutely agree that our study has several shortcomings being typical of many descriptive studies. The critique on the absence of a clear disease definition is correct. Due to the fact that our data were obtained from living subjects, no immunohistological staining of amyloid was possible, as it was also clearly discussed in the study [1]. In the same context, an unpleasant and partly invasive nerve conduction testing of the median nerve was not done because it should have been performed in all 385 participants, whether they had symptoms or not. Certainly, this test could have been applied only in patients having clinical signs because the combination of clinical symptoms and electrodiagnostic findings is the most valid way of diagnosing carpal tunnel syndrome (CTS) [2]. However, the outpatient nature of our study prohibited such design. It was not possible to examine all patients from the different dialysis centers by the same equipment and examiner which is a necessary prerequisite for correct diagnosis. Moreover, most patients declined additional diagnostic tests. It should be mentioned that the quoted reference reported only on CTS in HD if patients required operation $(0 \%<8$ years, $50 \%$ after 14 years and $100 \%$ after 20 years) [3]. Rates reporting clinical findings are expected to be higher. Moreover, in our study, the rate of patients who were already treated by operation was substantially higher (8.8\%). Especially in these cases, the diagnosis of CTS should have been previously confirmed and thus indicate the higher rate of CTS in our HD population. Nevertheless, we agree with the assumption that several cases of CTS might have been caused by diseases other than dialysis-related amyloidosis (DRA). But unfortunately, the main clinical symptoms of CTS in DRA are identical to those of idiopathic CTS, or CTS accompanying other diseases [3]. Since age is a strong predictor of CTS and DRA $[3,4]$, it should be taken into account that our dialysis population was substantially older (median 65.1 years) than in most other studies [3]. Moreover, several other known risk factors for CTS/ DRA could be confirmed in our trial [1]. The (paradoxical) findings concerning DRA and the use of high-flux dialyzers were adequately discussed in our paper [1].

\section{References}

1 Busch M, Schwenzky A, Franke S, Stein G, Wolf G: Advanced glycation end products and $\beta_{2}$-microglobulin as predictors of carpal tunnel syndrome in hemodialysis patients. Blood Purif 2012;34:3-9.

2 Aroori S, Spence RA: Carpal tunnel syndrome. Ulster Med J 2008;77:6-17.
The cross-sectional and retrospective character of our study implies the nonrandomized design. This includes the absence of information concerning the use and the quality of dialysis fluid in the past which is an obvious limitation of our study [1]. Prospective studies strictly using different types of dialyzers in different patient groups are welcome and would help to solve some of the open questions. Due to limitations regarding the length of our paper, these points could not be discussed in depth. The presented retrospective and currently unpublished observation in HD groups by Schiffl et al. showed a decrease in histologically proven CTS from $68 \%$ in the period $1990-2000$ to $28 \%$ in the period 2000-2010. Although details on these results are lacking, our clinical finding of $31.7 \%$ CTS cases seems not quite different from their results [1].

Besides discussion about rates, it should be noticed that our study was directed to the potential causes of CTS in HD like malnutrition or increased serum concentrations of $\beta_{2}$-microglobulin and advanced glycation end products.

3 Koch KM: Dialysis-related amyloidosis. Kidney Int 1992;41:1416-1429.

4 Stein G, Schneider A, Thoss K, Ritz E, Schaefer K, Huller M, Sperschneider H, Marzoll I: Beta 2-microglobulin serum concentration and associated amyloidosis in dialysis patients. Nephrol Dial Transplant 1991;6(suppl 3):57-61. 